University of Nebraska - Lincoln

DigitalCommons@University of Nebraska - Lincoln

Public Health Resources

Public Health Resources

2002

\title{
A preliminary linkage map of the tick, Ixodes scapularis
}

Amy J. Ullmann

Division of Vector-Borne Infectious Diseases, National Center for Infectious Diseases, Centers for Disease Control and Prevention, P.O. Box 2087, Rampart Road, Foothills Campus, Fort Collins, CO 80522, USA

Joseph Piesman

Division of Vector-Borne Infectious Diseases, National Center for Infectious Diseases, Centers for Disease Control and Prevention, P.O. Box 2087, Rampart Road, Foothills Campus, Fort Collins, CO 80522, USA

M.C. Dolan

Division of Vector-Borne Infectious Diseases, National Center for Infectious Diseases, Centers for Disease Control and Prevention, P.O. Box 2087, Rampart Road, Foothills Campus, Fort Collins, CO 80522, USA

William C. Black, IV

Department of Microbiology, Colorado State University, Fort Collins, CO 80523-1677, USA

Follow this and additional works at: https://digitalcommons.unl.edu/publichealthresources

Part of the Public Health Commons

Ullmann, Amy J.; Piesman, Joseph; Dolan, M.C.; and Black, IV, William C., "A preliminary linkage map of the tick, Ixodes scapularis" (2002). Public Health Resources. 114.

https://digitalcommons.unl.edu/publichealthresources/114

This Article is brought to you for free and open access by the Public Health Resources at DigitalCommons@University of Nebraska - Lincoln. It has been accepted for inclusion in Public Health Resources by an authorized administrator of DigitalCommons@University of Nebraska - Lincoln. 


\title{
A preliminary linkage map of the tick, Ixodes scapularis
}

\author{
AMY J. ULLMANN ${ }^{1, *}$, JOSEPH PIESMAN ${ }^{1}$, M.C. DOLAN ${ }^{1}$ and \\ WILLIAM C. BLACK, IV ${ }^{2}$ \\ ${ }^{1}$ Division of Vector-Borne Infectious Diseases, National Center for Infectious Diseases, \\ Centers for Disease Control and Prevention, P.O. Box 2087, Rampart Road, Foothills \\ Campus, Fort Collins, CO 80522, USA \\ ${ }^{2}$ Department of Microbiology, Colorado State University, Fort Collins, CO 80523-1677, USA
}

\begin{abstract}
A linkage map of the Ixodes scapularis genome was constructed based upon segregation amongst 127 loci. These included 84 random amplified polymorphic DNA (RAPD) markers, 32 Sequence-Tagged RAPD (STAR) markers, 5 cDNAs, and 5 microsatellites in 232 $\mathrm{F}_{1}$ intercross progeny from a single, field-collected $\mathrm{P}_{1}$ female. A preliminary linkage map of $616 \mathrm{cM}$ was generated across 14 linkage groups with one marker every $10.8 \mathrm{cM}$. Assuming a genome size of $\sim 10^{9} \mathrm{bp}$, the relationship of physical to genetic distance is $\sim 300 \mathrm{~kb} / \mathrm{cM}$ in the I. scapularis genome.
\end{abstract}

Key words: Ixodes scapularis, linkage map, microsatellites, RAPD-SSCP, STARs, cDNASSCP

\section{Introduction}

Ticks and the pathogens they transmit have long been an important cause of morbidity and mortality. Ticks are second only to mosquitoes as vectors of human and animal diseases (Sonenshine, 1991). Ixodes scapularis has become an important vector species because of its ability to transmit Borrelia burgdorferi, the causative agent of Lyme disease, in eastern and midwestern North America (Spielman et al., 1985). I. scapularis is also a vector of the causative agent of human granulocytic ehrlichiosis (HGE) (Telford et al., 1996) and Babesia microti the causative agent of human babesiosis (Spielman, 1976).

A great deal of information is known about the development, physiology, anatomy and vector competence of ticks, however little is understood about the genetic basis of these traits. Karyotypes have been characterized for 103

\footnotetext{
*Author for correspondence (E-mail: aff1@cdc.gov)
} 
of the $\sim 830$ known tick species (Oliver, 1977). However, a linkage map has never been produced for any of these or, for that matter, for any chelicerate arthropod. This is in part due to the minimal generation time required to establish a tick mapping family, which, at a minimum, over 1 year and 10 months are required.

Once an $\mathrm{F}_{1}$ intercross family has been established, the modern paradigm for linkage mapping involves simultaneous estimates of recombination amongst hundreds (Antolin et al., 1996) or potentially thousands (Yasukochi, 1998) of highly polymorphic loci. This typically yields an intensive linkage map with one marker distributed every $5 \mathrm{cM}$. Many different genetic markers are currently in use for intensive linkage mapping. One class includes simple sequence repeats or microsatellites (MS). MS-based linkage maps have been generated for anopheline mosquitoes (Zheng et al., 1996), mice (Dietrich et al., 1992) and humans (Hudson et al., 1995). Characterization of MS markers in I. scapularis has been described (Fagerberg et al., 2001) but the abundance of and heterozygosity at MS loci was generally low.

Random Amplified Polymorphic DNA amplified by the Polymerase Chain Reaction (RAPD-PCR) provides another class of highly polymorphic markers. This technique uses a short (10 oligonucleotide) primer to amplify small regions of a genome (Williams et al., 1990). Alleles amplified by RAPDPCR are typically dominant. Recessive alleles arise through mutations in or around the primer annealing site that cause the PCR process to fail. Genetic linkage maps utilizing RAPDs have been created for many insects including Anopheles gambiae (Dimopoulos et al., 1996), Aedes aegypti and a parasitic wasp, Bracon hebetor (Antolin et al., 1996), Apis mellifera (Hunt and Page, 1995), and Bombyx mori (Yasukochi, 1998). However, a great deal of the information needed to estimate recombination frequencies is lost when mapping loci with dominant alleles.

Single strand conformation polymorphism (SSCP) analysis of RAPD markers can reveal alternative, codominant alleles (Antolin et al., 1996). SSCP analysis is based on the principle that electrophoretic mobilities of single-strand DNA molecules in nondenaturing gels are dependent upon both the size and shape of the fragments. Several stable structures or conformations can be formed when secondary base pairing occurs among nucleotides on a single DNA strand. The SSCP technique detects $99-100 \%$ of point mutations in DNA molecules 100-300 base pairs (bp) in length and at least $89 \%$ of mutations in molecules 300-450 bp in length (Orita et al., 1989; Hayashi, 1991; Hiss et al., 1994; Vidal-Puig and Moller, 1994).

Sequence-Tagged RAPD loci (STARs) (Bosio et al., 2000) are another useful modification of RAPD markers. A RAPD-PCR band from a SSCP gel is cloned into a plasmid vector and sequenced. The sequence is then used to 
design targeted primers for PCR. STAR products are analyzed on a second SSCP gel, and usually segregate as codominant markers.

SSCP analysis of cDNA sequences provides another class of highly polymorphic markers for use in linkage mapping. Although these are unique, functional regions of a genome, polymorphisms are frequent, and can be used to generate linkage maps. This method has been used to map cDNA makers in A. aegypti (Fulton et al., 2001).

In this paper we describe the use of MS, RAPD-PCR, STAR, and cDNA markers to generate a preliminary linkage map in an $\mathrm{F}_{1}$ intercross family of $I$. scapularis. We describe our successes and failures in developing polymorphic markers and provide a preliminary $616 \mathrm{cM}$ linkage map across 14 linkage groups, the $1 \mathrm{~N}$ number of chromosomes determined cytogenetically (Oliver, 1977). This provided a linkage map with one marker every $10.8 \mathrm{cM}$. However, counting markers that did not map to any of these 14 linkage groups, we estimate a much larger map size of $3165 \mathrm{cM}$ with an average resolution of only one marker every $55 \mathrm{cM}$.

\section{Materials and Methods}

\section{Mapping cross}

All ticks were kept in Wheaton $8 \mathrm{ml}$ sample vials (Wheaton, Millville, NJ) which were placed into glass desiccator jars with water in the bottom to maintain a high relative humidity. The desiccators were maintained in a Revco bioclimatic chamber (Revco Inc., Deerfield, MI) at $21^{\circ} \mathrm{C}, 95 \%$ humidity and received a photoperiod of 16:8 (light:dark). ICR outbred, pathogen-free mice were used for blood feedings (DVBID, CDC, Ft. Collins). Four fertilized $\mathrm{P}_{1}$ females were collected from Bridgeport, Connecticut in the fall of 1997, brought back to DVBID, and fed on rabbits. Eggs were laid by the $\mathrm{P}_{1}$ females and hatched $\sim 4$ weeks later to generate four initial families. After eggs were collected from each $\mathrm{P}_{1}$ mother, each female was frozen in a labeled cryotube at $-20^{\circ} \mathrm{C}$ in $70 \%$ ethanol. $\mathrm{F}_{1}$ eggs from each female were hatched out and larvae were blood fed on mice. One hundred replete larvae were place in Wheaton vials and $\sim 5$ weeks after being fed, the larvae molted to nymphs. Once the cuticle hardened, the $F_{1}$ nymphs were blood fed. At this point the nymphs went into diapause for $\sim 8$ months due to an unintentional drop in incubator temperature to $16^{\circ} \mathrm{C}$. When the incubator temperature was corrected, the ticks came out of diapause and development continued. When the fed nymphs molted to adults, eight single $F_{1}$ intercrosses were set up per family. Successful breeding pairs were established from $\mathrm{P}_{1}$ 
female \#2 (five pairs), and only a single pair from $\mathrm{P}_{1}$ females \#1, \#3, and $\# 4$. For each $F_{1}$ intercross, females were isolated from the males, and single breeding pairs were made and subsequently fed individually in an ear bag on a rabbit ear.

Fed females were separated into labeled vials to collect $F_{2}$ eggs. These were laid from 4 to 6 weeks post-feeding. Each $F_{1}$ male was frozen in a labeled tube at $-20^{\circ} \mathrm{C}$ in $70 \%$ ethanol, and given the same number as his $\mathrm{F}_{1}$ mate. Each $F_{1}$ female was frozen after collecting eggs. $F_{2}$ eggs were hatched and when the cuticle had hardened, blood fed on mice. After the larvae had molted to nymphs, $\sim 4$ weeks post-feed, and the cuticle had hardened, nymphs were fed on mice. The nymphs were reared to adults, and individually assigned a number that corresponded to the $P_{1}$ and $F_{1}$ female numbers. The $F_{2}$ adults were then frozen in a labeled tube at $-20^{\circ} \mathrm{C}$ in $70 \%$ ethanol.

\section{Markers}

Microsatellite loci were derived as described by Fagerberg et al. (2001). RAPD loci were amplified and run on SSCP gels as described in Black and DuTeau (1997). STAR loci were derived from polymorphic RAPD loci following exactly the procedure in Bosio et al. (2000). Primers for cDNA sequences from an I. scapularis salivary gland genomic library (kindly provided by Jose Ribeiro) were developed following the methodology of Fulton et al. (2001).

\section{Map data analysis}

Map distances were converted from recombination fractions to map units (cM) using the Kosambi mapping function (Kosambi, 1944). Offspring genotypes of $F_{1}$ and $F_{2}$ I. scapularis were entered into JoinMap ${ }^{\circledR} 2.0$ (Stam and van Oojin, 1995). Because the numbers of parental, recombinant, and uninformative genotypes, and the segregation ratios among the $F_{2}$ offspring may differ for each locus, JoinMap allows each marker to be analyzed according to parental genotypes. Initially, a threshold recombination fraction of 0.499 and a log odds density (LOD) score of 3.0 was used to group markers. The minimal LOD was then increased to 6.0 in increments of 0.1 to monitor the rate at which markers left individual linkage groups. Increasing the minimum LOD in increments tests how robust are the linkage groups in the genotype data set. DrawMap ${ }^{\circledR}$ (van Ooijen, 1994) was used to plot a linkage map from the recombination frequencies generated by JoinMap. 


\section{Results}

The $\mathrm{P}_{1}$ generation began as a field-collected, fertilized female. The genotype of the $\mathrm{P}_{1}$ male had therefore to be inferred at all loci. Recombination frequencies among all marker loci were analyzed in $232 \mathrm{~F}_{2}$ progeny.

Twenty RAPD primers (Table 1) amplified 84 polymorphic marker loci. Genotypes at 64 loci were in expected Mendelian ratios among the $F_{2}$ offspring. Sixty-three of these loci and D04.800a and b (bands $\sim 1 \mathrm{~mm}$ apart on the SSCP gel that did not completely cosegregate) were extracted from SSCP gels, reamplified, cloned and sequenced to generate 65 primer pairs (Table 2 ). Of these, 52 STAR loci were polymorphic, and genotypes at 33 of these were in expected Mendelian ratios in the $\mathrm{F}_{2}$ offspring. Alleles at 20 of the 33 STAR loci segregated as codominant markers.

Fifty-six sequences from an I. scapularis salivary gland cDNA library (sequences kindly provided by Jose Ribeiro) were analyzed for primer design.

Table 1. List of RAPD primers and sequences utilized for mapping

\begin{tabular}{ll}
\hline Primer & Sequence \\
\hline A09 & GGGTAACGCC \\
A20 & GTTGCGATCC \\
B15 & GGAGGGTGTT \\
B18 & CCACAGCAGT \\
B20 & GGACCCTTAC \\
C19 & GTTGCCAGCC \\
C01 & TTCGAGCCAG \\
C04 & CCGCATCTAC \\
D02 & GGACCCAACC \\
D03 & GTCGCCGTCA \\
D04 & TCTGGTGAGG \\
D07 & TTGGCACGGG \\
D08 & GTGTGCCCCA \\
D12 & CACCGTATCC \\
D13 & GGGGTGAGCA \\
D16 & AGGGCGTAAG \\
D17 & TTTCCCACGG \\
D18 & GAGAGCCAAC \\
D19 & CTGGGGACTT \\
D20 & ACCCGGTCAC \\
\hline &
\end{tabular}


Table 2. List of 95 primer pairs for PCR amplification of 65 STAR, 20 cDNA and 10 microsatellite loci in I. scapularis

\begin{tabular}{|c|c|c|c|c|}
\hline Locus name & & $\mathrm{T}_{\mathrm{a}}$ & Length (bp) & Primer sequences \\
\hline \multicolumn{5}{|l|}{ STARs } \\
\hline A09.306ST & Genbank Acc. \#BZ385505 & 49 & 306 & $\begin{array}{l}\text { GGGTAACGCCAGGGTTTTCC } \\
\text { GGGTAACGCCCGATGTATAG }\end{array}$ \\
\hline A20.310ST & Genbank Acc. \#BZ592381 & 51 & 310 & $\begin{array}{l}\text { GTTGCGATCCCTCAGAGCGA } \\
\text { GTTGCGATCCAACGAAGTTT }\end{array}$ \\
\hline A20.390ST & Genbank Acc. \#BZ592382 & 57 & 390 & $\begin{array}{l}\text { GTTGCGATCCCAGGATATAC } \\
\text { GTTGCGATCCTASAGCACAT }\end{array}$ \\
\hline $\mathrm{A} 20.517 \mathrm{ST}$ & Genbank Acc. \#BZ592383 & 59 & 517 & $\begin{array}{l}\text { GTTGCGATCCCTGCGCCTAT } \\
\text { GTTGCGATCCAGGCGATCAC }\end{array}$ \\
\hline $\mathrm{B} 15.874 \mathrm{ST}$ & Genbank Acc. \#BZ385506 & 59 & 874 & $\begin{array}{l}\text { GGAGGGTGTTGCACAGTACA } \\
\text { GGAGGGTGTTGGGGTGTGTG }\end{array}$ \\
\hline $\mathrm{B} 18.358 \mathrm{ST}$ & Genbank Acc. \#BZ385507 & 62 & 358 & $\begin{array}{l}\text { CCACAGCAGTCAAACCTTCT } \\
\text { CCACAGCAGTAGTGATACTC }\end{array}$ \\
\hline $\mathrm{B} 18.653 \mathrm{ST}$ & Genbank Acc. \#BZ385508 & 62 & 653 & $\begin{array}{l}\text { CCACAGCAGTCGACCATGCG } \\
\text { CCACAGCAGTAGTGATACTC }\end{array}$ \\
\hline B20.361ST & Genbank Acc. \#BZ385509 & 62 & 361 & $\begin{array}{l}\text { GGACCCTTACGAGCGAAAGA } \\
\text { GGACCCTTACCCCAACCAAA }\end{array}$ \\
\hline C13.357ST & Genbank Acc. \#BZ385510 & 62 & 357 & $\begin{array}{l}\text { AAGCCTCGTCGGGGTAGAGA } \\
\text { AAGCCTCGTCCTGCTGTTCT }\end{array}$ \\
\hline C13.579ST & Genbank Acc. \#BZ385511 & 60 & 579 & $\begin{array}{l}\text { AAGCCTCGTCGTTAGACGTT } \\
\text { AAGCCTCGTCCACTTTCCTT }\end{array}$ \\
\hline $\mathrm{C} 16.362 \mathrm{ST}$ & Genbank Acc. \#BZ3855512 & 62 & 362 & $\begin{array}{l}\text { CACACTCCAGTCAGCATCAG } \\
\text { CACACTCCAGGACCAATAGT }\end{array}$ \\
\hline
\end{tabular}




\begin{tabular}{|c|c|c|c|c|}
\hline C16.432ST & Genbank Acc. \#BZ385513 & 46 & 432 & $\begin{array}{l}\text { CACACTCCAGGCAAAAAAAC } \\
\text { CACACTCCAGCATTAGGGAA }\end{array}$ \\
\hline \multirow[t]{2}{*}{ C16.458ST } & Genbank Acc. \#BZ385514 & 59 & 458 & CACACTCCAGGCAGATGCAT \\
\hline & & & & CACACTCCAGCATCAAGTGC \\
\hline \multirow[t]{2}{*}{ C16.680ST } & Genbank Acc. \#BZ385515 & 48 & 680 & САСАСТССАССААТССССТА \\
\hline & & & & CACACTCCACGATACAAACG \\
\hline \multirow[t]{2}{*}{ C01.169ST } & Genbank Acc. \#BZ385516 & 59 & 169 & TTCGAGCCAGGGCAGGACCT \\
\hline & & & & TTCGAGCCAGCATTGATAAG \\
\hline \multirow[t]{2}{*}{ C01.170ST } & Genbank Acc. \#BZ385517 & 59 & 170 & TTCGAGCCAGCATTGAGAAG \\
\hline & & & & TTCGAGCCAGGGCAGGACCT \\
\hline \multirow[t]{2}{*}{ C04.323ST } & Genbank Acc. \#BZ385518 & 60 & 323 & CCGCATCTACGTACGATTGA \\
\hline & & & & CCGCATCTACGCCCGGTAGT \\
\hline \multirow[t]{2}{*}{ C04.331ST } & Genbank Acc. \#BZ385519 & 57 & 331 & CCGCATCTACATATACAATA \\
\hline & & & & CCGCATCTACCCCATCGACG \\
\hline \multirow[t]{2}{*}{ C04.345ST } & Genbank Acc. \#BZ385520 & 57 & 345 & CCGCATCTACGTTAGCAGTC \\
\hline & & & & CCGCATCTACACACAGTTTC \\
\hline \multirow[t]{2}{*}{ D02.328ST } & Genbank Acc. \#BZ385392 & 50 & 328 & GGACCCAACCCTATACCGCT \\
\hline & & & & GGACCCAACCTACGGAGGAG \\
\hline \multirow[t]{2}{*}{ D02.330ST } & Genbank Acc. \#BZ385393 & 48 & 330 & GGACCCAACCCTATACCGCT \\
\hline & & & & GGACCCAACCTACGGAGGAG \\
\hline \multirow[t]{2}{*}{ D02.460ST } & Genbank Acc. \#BZ385394 & 45 & 460 & GGACCCAACCATGTTTTAAA \\
\hline & & & & GGACCCAACCCCAAGAAATC \\
\hline \multirow[t]{2}{*}{ D02.463ST } & Genbank Acc. \#BZ385395 & 48 & 463 & GGACCCAACCCCCAAGAATC \\
\hline & & & & GGACCCAACCATGTTTTAAC \\
\hline
\end{tabular}


Table 2. (continued)

\begin{tabular}{|c|c|c|c|c|}
\hline Locus name & & $\mathrm{T}_{\mathrm{a}}$ & Length (bp) & Primer sequences \\
\hline $\mathrm{D} 02.464 \mathrm{ST}$ & Genbank Acc. \#BZ385396 & 48 & 464 & $\begin{array}{l}\text { GGACCCAACCCCAAGAATCC } \\
\text { GGACCCAACCATGTTTTAAC }\end{array}$ \\
\hline D02.466ST & Genbank Acc. \#BZ385397 & 48 & 466 & $\begin{array}{l}\text { GGACCCAACCCCAAGAAATC } \\
\text { GGACCCAACCATGTTTTAAC }\end{array}$ \\
\hline D02.470ST & Genbank Acc. \#BZ385398 & 50 & 470 & $\begin{array}{l}\text { GGACCCAACCATGTTTTAAC } \\
\text { GGACCCAACCCCCAAGAATC }\end{array}$ \\
\hline D02.472ST & Genbank Acc. \#BZ385399 & 48 & 472 & $\begin{array}{l}\text { GGACCCAACCATGTTTTAAA } \\
\text { GGACCCAACCCCAAGAATCC }\end{array}$ \\
\hline D04.410ST & Genbank Acc. \#BZ385400 & 45 & 410 & $\begin{array}{l}\text { TCTGGTGAGGGGGAAGAAGG } \\
\text { TCTGGTGAGGCAACTACTGA }\end{array}$ \\
\hline D04.534ST & Genbank Acc. \#BZ385401 & 45 & 534 & $\begin{array}{l}\text { TCTGGTGAGGCGTTCAAGGC } \\
\text { TCTGGTGAGGAGGAATGTTC }\end{array}$ \\
\hline D04.800AST & $\begin{array}{l}\text { Genbank Acc. \#BZ85433- } \\
\text { BZ85434 }\end{array}$ & 45 & 800 & $\begin{array}{l}\text { TCTGGTGAGGTTCGTGTCAA } \\
\text { TCTGGTGAGGCGGGGCTCCA }\end{array}$ \\
\hline D04.800BST & $\begin{array}{l}\text { Genbank Acc. \#BZ85435- } \\
\text { BZ85436 }\end{array}$ & 45 & 800 & $\begin{array}{l}\text { TCTGGTGAGGCGGGACTTCA } \\
\text { TCTGGTGAGGTTCGTGTCAA }\end{array}$ \\
\hline D07.292ST & Genbank Acc. \#BZ385402 & 45 & 292 & $\begin{array}{l}\text { TTGGCACGGGCACACAAATA } \\
\text { TTGGCACGGGGCTGCAACAA }\end{array}$ \\
\hline D07.434ST & Genbank Acc. \#BZ385403 & 50 & 434 & $\begin{array}{l}\text { TTGGCACGGGCAATCATTGC } \\
\text { TTGGCACGGGACGCGGAAGC }\end{array}$ \\
\hline
\end{tabular}




\begin{tabular}{|c|c|c|c|c|}
\hline D07.457ST & Genbank Acc. \#BZ385404 & 50 & 457 & $\begin{array}{l}\text { TTGGCACGGGCGCGTGGCTT } \\
\text { TTGGCACGGGGGACAAAAGA }\end{array}$ \\
\hline \multirow[t]{2}{*}{ D07.649ST } & Genbank Acc. \#BZ385405 & 50 & 649 & TTGGCACGGGCAGCAGCCGG \\
\hline & & & & TTGGCACGGGTGGAGGTCGT \\
\hline \multirow[t]{2}{*}{ D08.459ST } & Genbank Acc. \#BZ385406 & 50 & 459 & GTGTGCCCCAGGACCTGGGT \\
\hline & & & & GTGTGCCCCACCGCAAAGAG \\
\hline \multirow[t]{2}{*}{ D08.463ST } & Genbank Acc. \#BZ385407 & 50 & 463 & GTGTGCCCCACCGCAAAGAG \\
\hline & & & & GTGTGCCCCAGGACCTGGGT \\
\hline \multirow[t]{2}{*}{ D12.425ST } & Genbank Acc. \#BZ385408 & 50 & 425 & CACCGTATCCAGGAAGTGTT \\
\hline & & & & CACCGTATCCTCGCAGAGTT \\
\hline \multirow[t]{2}{*}{ D12.617ST } & Genbank Acc. \#BZ385409 & 36 & 617 & CACCGTATCCCCCTAGCGAA \\
\hline & & & & CACCGTATCCAACAAAGGAT \\
\hline \multirow[t]{2}{*}{$\mathrm{D} 12.821 \mathrm{ST}$} & Genbank Acc. \#BZ385410 & 43 & 821 & CACCGTATCCCGTTGGCCTA \\
\hline & & & & CACCGTATCCCCATGATTTC \\
\hline \multirow[t]{2}{*}{ D12.942ST } & Genbank Acc. \#BZ385411 & 50 & 942 & CACCGTATCCATGTGTAATC \\
\hline & & & & CACCGTATCCCCAGACTCGG \\
\hline \multirow[t]{2}{*}{ D17.459ST } & Genbank Acc. \#BZ385412 & 45 & 459 & TTTCCCACGGGCACGTACTA \\
\hline & & & & TTTCCCACGGCGTCATGACT \\
\hline \multirow[t]{2}{*}{ D17.984ST } & Genbank Acc. \#BZ385413 & 48 & 984 & TTTCCCACGGCAGGATAACT \\
\hline & & & & TTTCCCACGGATTAATCAAA \\
\hline \multirow[t]{2}{*}{ D17.1200ST } & Genbank Acc. \#BZ385437- & 43 & 1200 & TTTCCCACGGAACGACTCAC \\
\hline & BZ85438 & & & TTTCCCACGGCCAAACAAAG \\
\hline \multirow[t]{2}{*}{ D13.363ST } & Genbank Acc. \#BZ385414 & 50 & 363 & GGGGTGACGACCGACTGGC \\
\hline & & & & GGGGTGACGAGACAACGAAA \\
\hline
\end{tabular}




\begin{tabular}{|c|c|c|c|c|}
\hline Locus name & & $\mathrm{T}_{\mathrm{a}}$ & Length (bp) & Primer sequences \\
\hline D13.443ST & Genbank Acc. \#BZ385415 & 50 & 443 & $\begin{array}{l}\text { GGGGTGACGATGGGTTGTGG } \\
\text { GGGGTGACGACCAACCCGGG }\end{array}$ \\
\hline D16.330ST & Genbank Acc. \#BZ385416 & 50 & 330 & $\begin{array}{l}\text { AGGGCGTAAGCAGAACCGTT } \\
\text { AGGGCGTAAGCATCAGGTAT }\end{array}$ \\
\hline D16.470ST & Genbank Acc. \#BZ385417 & 50 & 470 & $\begin{array}{l}\text { AGGGCGTAAGGGCCGATAGC } \\
\text { AGGGCGTAAGTGCGTGTGTG }\end{array}$ \\
\hline D16.521ST & Genbank Acc. \#BZ385418 & 50 & 521 & $\begin{array}{l}\text { AGGGCGTAAGCCTAACAAAA } \\
\text { AGGGCGTAAGGGTAGTGTGT }\end{array}$ \\
\hline D17.684ST & Genbank Acc. \#BZ385419 & 50 & 684 & $\begin{array}{l}\text { TTTCCCACGGCACCCTTTGT } \\
\text { TTTCCCACGGGCCATTCCGC }\end{array}$ \\
\hline $\mathrm{D} 18.265 \mathrm{ST}$ & Genbank Acc. \#BZ385420 & 41 & 265 & $\begin{array}{l}\text { GAGAGCCAACACGTCAAATA } \\
\text { GAGAGCCAACCTCACTGACC }\end{array}$ \\
\hline D18.266ST & Genbank Acc. \#BZ385421 & 43 & 266 & $\begin{array}{l}\text { GAGAGCCAACCTCACTGACCT } \\
\text { GAGAGCCAACACGTCAAAT }\end{array}$ \\
\hline D18.284ST & Genbank Acc. \#BZ385422 & 39 & 284 & $\begin{array}{l}\text { GAGAGCCAACCTACTTGGCA } \\
\text { GAGAGCCAACATGGAGAGGA }\end{array}$ \\
\hline D18.353ST & Genbank Acc. \#BZ385423 & 39 & 353 & $\begin{array}{l}\text { GAGAGCCAACGGGTAAATAG } \\
\text { GAGAGCCAACAAGAGACTAC }\end{array}$ \\
\hline D18.366ST & Genbank Acc. \#BZ385424 & 50 & 366 & $\begin{array}{l}\text { GAGAGCCAACCCACCTTCAT } \\
\text { GAGAGCCAACGCGCGGGCGA }\end{array}$ \\
\hline D18.367ST & Genbank Acc. \#BZ385425 & 50 & 367 & $\begin{array}{l}\text { GAGAGCCAACGCGCGGGCGA } \\
\text { GAGAGCCAACCCACCTACAT }\end{array}$ \\
\hline
\end{tabular}




\section{D18.369ST}

D18.826ST

D19.382ST

D19.833ST

D20.284ST

D20.294ST

D20.817ST

\section{$\mathrm{cDNA}^{\mathrm{a}}$}

Is218 (APOP-IN

(AF333765) DAD-1-like

protein [Schistosoma

japonicum] $\left.(3.00 \mathrm{E}-41)^{*}\right)$

Is155 (ATP-S

(mitochondrial ATP synthase

[Drosophila melanogaster]

(1.00E-25))
Genbank Acc. \#BZ385426

Genbank Acc. \#BZ385427

Genbank Acc. \#BZ385428

Genbank Acc. \#BZ385429

Genbank Acc. \#BZ385430

Genbank Acc. \#BZ385431

Genbank Acc. \#BZ385432

Genbank Acc. \#CA76376

Genbank Acc. \#CA763762
50

369

50

50

50

60

209

52
GCCAGCGTAAACGAAACC

GATGAAGCCCGACAGGAA

GAGAGCCAACCCACCTTCAT

GAGAGCCAACCCGCGGGCGA

GAGAGCCAACAATCAACCTC

GAGAGCCAACGCCAAAGAAA CTGGGGACTTCAAACAACCC

CTGGGGACTTGCCACGTCAG

CTGGGGACTTCACAGAGGCA

CTGGGGACTTCCGTTTGGAT ACCCGGTCACCAATTTGTAG ACCCGGTCACAGATGGAGAA ACCCGGTCACACGAGCGCTT ACCCGGTCACGTCTTCACTG ACCCGGTCACCAGCAGATTT ACCCGGTCACATCATTGTGT

TTGAAAGGGTCCCAGAAGA GGAATGGCACCGTAAAGC 


\begin{tabular}{|c|c|c|c|c|}
\hline Locus name & & $\mathrm{T}_{\mathrm{a}}$ & Length (bp) & Primer sequences \\
\hline $\begin{array}{l}\text { Is108 (EF1-A } \\
\text { (AF378368 elongation factor } \\
\text { 1-alpha [Coccidioides } \\
\text { immitis] (3.00E-34)) }\end{array}$ & Genbank Acc. \#CA763763 & 62 & 196 & $\begin{array}{l}\text { TGTGGGCGTCATCAAGTC } \\
\text { AAACAGAGTAACCAAACGAAAG }\end{array}$ \\
\hline $\begin{array}{l}\text { Is } 119 \text { (ELF-2B } \\
\text { (CAC08449 eukaryote initiation } \\
\text { factor } 2 \text { beta [Gallus gallus] } \\
(5.00 \mathrm{E}-57))\end{array}$ & Genbank Acc. \#CA763764 & 58 & 301 & $\begin{array}{l}\text { CCTTCGCCAACTTCCTCG } \\
\text { AAGCCAGACTTGATGCTCAC }\end{array}$ \\
\hline $\begin{array}{l}\text { Is211 (GST-1 } \\
\text { (AF366931 glutathione } \\
\text { S-transferase [Boophilus } \\
\text { microplus]) }(1.00 \mathrm{E}-30))\end{array}$ & Genbank Acc. \#CA763765 & 53 & 218 & $\begin{array}{l}\text { GAAATAACGGTTGAGGGC } \\
\text { GTAGGGAAGGTTGGGAAA }\end{array}$ \\
\hline $\begin{array}{l}\text { Is75 (GST-2 (JX0095 } \\
\text { glutathione transferase } \\
\quad(\text { EC } 2.5 .1 .18) b-\text { guinea pig) } \\
\quad(1.00 \mathrm{E}-30))\end{array}$ & Genbank Acc. \#CA763766 & 53 & 164 & $\begin{array}{l}\text { TCCTCGTTGAGATCCAGT } \\
\text { GTTCCAGCAGTAATAGTCG }\end{array}$ \\
\hline $\begin{array}{l}\text { ISAC (AF270496 I. } \\
\text { scapularis anticomplement } \\
\text { protein) }\end{array}$ & & 56 & 188 & $\begin{array}{l}\text { TTTTGGCGATTTCGTTTC } \\
\text { AATTCCTTCCCAGGTTGC }\end{array}$ \\
\hline $\begin{array}{l}\text { Is } 152 \text { (ND(CG7580 } \\
\text { gene product }[D . \\
\text { melanogaster }] \text { putative NADH } \\
\text { dehydrogenase) }(6.00 \mathrm{E}-27))\end{array}$ & Genbank Acc. \#CA763767 & 53 & 251 & $\begin{array}{l}\text { GCACTTTGGGAACCTGAT } \\
\text { TGGGCTGCTTCTACTTGTC }\end{array}$ \\
\hline
\end{tabular}




\begin{tabular}{|c|c|c|c|c|}
\hline $\begin{array}{l}\text { Is136 (QM (XP } 141003 \\
\text { similar to ribosomal protein } \\
\text { L10 [Rattus norvegicus]) } \\
(2.00 \mathrm{E}-49))\end{array}$ & Genbank Acc. \#CA763768 & 62 & 184 & $\begin{array}{l}\text { AGGCCAAGGTGGATGAGTT } \\
\text { TTGTTGATGCGGATGACG }\end{array}$ \\
\hline $\begin{array}{l}\text { Is30 (RPS12 (AF470687 } \\
\text { 40S ribosomal } \\
\text { protein S12 [Branchiostoma } \\
\text { belcheri]) }(1.00 \mathrm{E}-49))\end{array}$ & Genbank Acc. \#CA763769 & 62 & 214 & $\begin{array}{l}\text { GCTCATCTGTGCGTCCTG } \\
\text { TCTCCTTGCCGTAGTCCTT }\end{array}$ \\
\hline $\begin{array}{l}\text { Is140 (RPS13 } \\
\begin{array}{l}\text { (AF116857 40S ribosomal } \\
\text { protein S13 [Cricetulus } \\
\text { griseus] })(3.00 \mathrm{E}-48))\end{array}\end{array}$ & Genbank Acc. \#CA763770 & 60 & 177 & $\begin{array}{l}\text { CCCAGTCGGCTCTGCCATAC } \\
\text { TCTTGTTGCCCGTCACCC }\end{array}$ \\
\hline $\begin{array}{l}\text { Is45 (RPS14B } \\
\text { (AF402822 40S ribosomal } \\
\text { protein S14 [Ictalurus } \\
\text { punctatus]) (3.00E-52)) }\end{array}$ & Genbank Acc. \#CA763771 & 53 & 183 & $\begin{array}{l}\text { TTTGGCGTAGCCCACATA } \\
\text { GACCTGCTTGCATTTCTCG }\end{array}$ \\
\hline $\begin{array}{l}\text { Is10 (RPS18) } \\
\text { (AAN52390 ribosomal } \\
\text { protein S18 [B. belcheri }] \text { ) } \\
(1.00 \mathrm{E}-65))\end{array}$ & Genbank Acc. \#CA763772 & 55 & 206 & $\begin{array}{l}\text { TCGCCCTGACAGCCATTA } \\
\text { CTGAACTTGCCGTCCTTG }\end{array}$ \\
\hline $\begin{array}{l}\text { Is66 (RPL12 } \\
\text { (AAH08230 ribosomal } \\
\text { protein L12 [Homo sapiens]) } \\
(1.00 \mathrm{E}-49))\end{array}$ & Genbank Acc. \#CA763773 & 61 & 310 & $\begin{array}{l}\text { CCCAAGTTTGATCCCACG } \\
\text { ATGTCAGGTTGCCGCTGT }\end{array}$ \\
\hline
\end{tabular}




\begin{tabular}{|c|c|c|c|c|}
\hline Locus name & & $\mathrm{T}_{\mathrm{a}}$ & Length (bp) & Primer sequences \\
\hline $\begin{array}{l}\text { Is149 (RPL17A) } \\
\text { (AF395586 ribosomal protein } \\
\text { L17/23 [Spodoptera frugiperda]) } \\
(1.00 \mathrm{E}-57))\end{array}$ & Genbank Acc. \#CA763774 & 44 & 293 & $\begin{array}{l}\text { GCATCTCCCTCGGTCTTC } \\
\text { ATTTCGCCTTTGTTGTTGA }\end{array}$ \\
\hline $\begin{array}{l}\text { Is171 (RPL40Ribosomal } \\
\text { Protein L40 ubiquitin } \\
\text { extension protein [D. melanogaster }] \\
(2.00 \mathrm{E}-41))\end{array}$ & Genbank Acc. \#CA763775 & 68 & 154 & $\begin{array}{l}\text { GACTGGTGGGTGGTGTCATT } \\
\text { GGTCGCAGGTTGTTGGAG }\end{array}$ \\
\hline $\begin{array}{l}\text { Is28 (RPL44) } \\
\text { (AAM94276 ribosomal } \\
\text { protein L44 [Chlamys } \\
\text { farreri] })(3.00 \mathrm{E}-49))\end{array}$ & Genbank Acc. \#CA763776 & 45 & 250 & $\begin{array}{l}\text { ATGATAAGAAGGGCAAGGAT } \\
\text { CTGGTGGATTAGAACTGGAT }\end{array}$ \\
\hline $\begin{array}{l}\text { Is27 (PRP } \\
\quad(\mathrm{AF} 400199 \text { ribosomal } \\
\text { protein L37 [S. frugiperda] } \\
(6.00 \mathrm{E}-30))\end{array}$ & Genbank Acc. \#CA763777 & 58 & 152 & $\begin{array}{l}\text { GCAGCTACCACATCCAAA } \\
\text { CTGAAACGCCTCCAGACC }\end{array}$ \\
\hline $\begin{array}{l}\text { SALP16 (AF061845 } \\
\text { Ixodes scapularis salivary } \\
\text { gland } 16 \mathrm{kDa} \text { protein } \\
\text { (salp16)) }\end{array}$ & & 63 & 371 & $\begin{array}{l}\text { CAGTGAGACGGGAGCATC } \\
\text { GTTCGGCACCTTCCTTTA }\end{array}$ \\
\hline $\begin{array}{l}\text { Is79 (UBQ (CAA44453 } \\
\text { ubiquitin-conjugating enzyme } \\
\quad[\text { D. melanogaster }](2.00 \mathrm{E}-43))\end{array}$ & Genbank Acc. \#CA763778 & 47 & 196 & $\begin{array}{l}\text { ACAGCAATGGCAGCATCT } \\
\text { TTCCTGGTCCACTCCCTA }\end{array}$ \\
\hline
\end{tabular}




\begin{tabular}{|c|c|c|c|c|}
\hline \multicolumn{5}{|l|}{ Microsatellites } \\
\hline \multirow[t]{2}{*}{ ISAG4 } & Genbank Acc. \#AF331743 & 41 & 250 & AAGAAAATAAAGCGAACAAG \\
\hline & & & & ATAAGCAATTCATACGAGATAGT \\
\hline \multirow[t]{2}{*}{ ISAG25 } & Genbank Acc. \#AF331742 & 57 & 171 & AAATGTCCGAACAGCCTTAT \\
\hline & & & & GCCCTTGAGTCTACCCACTA \\
\hline \multirow[t]{2}{*}{ ISAC4 } & Genbank Acc. \#AF331736-AF331739 & 49 & 170 & GGGTCCCAACGATTGCTAAACCAG \\
\hline & & & & AAGCGTATCCGATTTGCCCTTCAT \\
\hline \multirow[t]{2}{*}{ ISAC8 } & Genbank Acc. \#AF331740 & 42 & 170 & TCTTCCCGCTGCTGTCTCGTATTC \\
\hline & & & & GAGCTACCCCTTTCATCGTCTTCG \\
\hline \multirow[t]{2}{*}{ ISAC20 } & Genbank Acc. \#AF331734 & 42 & 150 & AGAAACACGGAAGGAGAAAGGAGA \\
\hline & & & & AACTGTGCCAGATGGGAAAGAAGA \\
\hline \multirow[t]{2}{*}{ ISAC22 } & Genbank Acc. \#AF331735 & 44 & 200 & CAGCTGGGCCCСТCCTTTTAATCC \\
\hline & & & & TATTGTAAGGCCAGTCGCCGCTGC \\
\hline \multirow[t]{2}{*}{ ISCAG12 } & Genbank Acc. \#AF331755 & 44 & 170 & GAAGAACACCGAGCGAACCAGAAC \\
\hline & & & & GTGAGCTGAGGGTTGCTGTTGATG \\
\hline \multirow[t]{2}{*}{ ISGATA3 } & Genbank Acc. \#AF331752 & 47 & 150 & AGTCCCCTCAGAGCGATTTTCA \\
\hline & & & & GGCCGCCAGTTTGATGGATA \\
\hline \multirow[t]{2}{*}{ ISGATA4 } & Genbank Acc. \#AF331753 & 41 & 200 & CAGACAATGTCATTCAATCGCA \\
\hline & & & & CGCACAATGCAAAACAAATCTA \\
\hline \multirow[t]{2}{*}{ ISCTGY17 } & Genbank Acc. \#AF331745-AF331747 & 41 & 150 & TTCTTGTTTTATTGGTTGGGTG \\
\hline & & & & AATGCAGGGTAAGTTGAGATTG \\
\hline
\end{tabular}

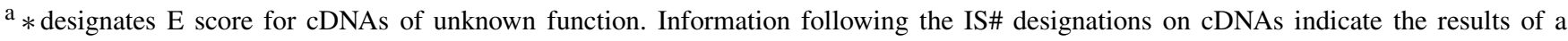
BLASTX search on Genbank. 
Primers were designed to amplify 250-400 bp fragments (optimal size for SSCP) in 20 of the genes (Table 2) with high matches to existing genes. However, ultimately only five of these could be mapped because the genomic DNA amplified by the primers was much larger than the anticipated size based upon the cDNA sequence. Amplified products from these loci were extracted from the polyacrylamide gels, reamplified and cloned into a plasmid vector for subsequent sequencing. As expected we found that the cDNA sequence was interrupted by large introns, some up to $800 \mathrm{bp}$ in size. SSCP analysis can only reliably reveal polymorphisms in fragments $<500 \mathrm{bp}$. We did not design internal primers from the genomic sequences.

Primers for 10 MS loci (Fagerberg et al., 2001) are also listed in Table 2. However, only six of these could be mapped, one of which was not in expected Mendelian ratios, consequently only five MS markers have been mapped. The remaining four were monomorphic, further supporting the observations of low heterozygosity found at I. scapularis MS loci (Fagerberg et al., 2001).

A linkage map was generated using JoinMap (Figure 1) (Ullmann et al., 2003). The linkage map contains only those loci at which genotypes were in Mendelian ratios. Fourteen linkage groups were identified by JMGRP at an LOD of 3.0. Above this LOD, markers began splitting away either singly or into separate groups. A total of 57 markers mapped across $616 \mathrm{cM}$ on the 14 linkage groups with one marker every $10.8 \mathrm{cM}$. A total map distance of $3166 \mathrm{cM}$ was estimated by making the assumption that the remaining 51 markers that did not cosegregate onto any of the 14 linkage groups were $\geq 50 \mathrm{cM}$ from mapped markers $(51$ markers $\times 50 \mathrm{cM} /$ marker $)+616 \mathrm{cM}=$ $3166 \mathrm{cM})$.

Linkage groups on the map are temporarily assigned numbers according to their relative lengths. These lengths could change if more markers are added to the map. Ultimately numbers will be assigned to chromosomes via physical mapping when a physical map for I. scapularis exists. When this is accomplished then chromosome numbers will be assigned according to overall chromosome length. Currently, the map shows that linkage group 1 is four times longer than any of the other 13 linkage groups. We suspect that linkage group 1 corresponds to the sex chromosome, because it is known from cytogenetic work that in Ixodes the sex chromosome is three to four times longer than the autosomes (Oliver, 1977), and when sex was mapped it was most closely linked to A09.583 on linkage group 1 with an LOD of 2.667.

The size of this linkage maps suggests a very high recombination rate for the I. scapularis genome. The physical size of another ixodid tick genome, Amblyomma americanum, was previously determined to be $1.08 \mathrm{pg}$ or $1.04 \times$ $10^{9} \mathrm{bp}$, and repetitive elements were in a long-period interspersion pattern (Palmer et al., 1994). Assuming this is an accurate estimate of the size of the 

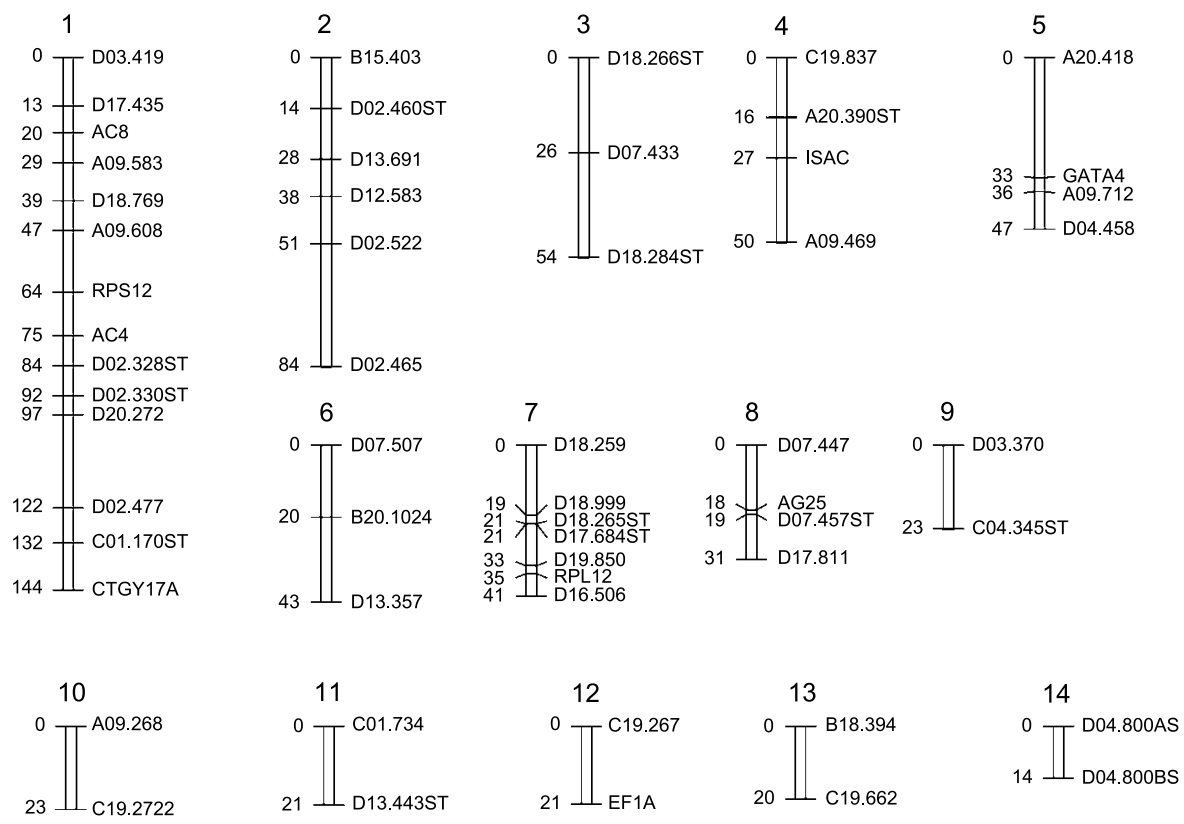

Figure 1. A preliminary linkage map of the I. scapularis genome that includes only those loci at which genotypes were in Mendelian ratios. Fourteen linkage groups were identified by JMGRP at an LOD of 3.0. A total of 57 markers map across $616 \mathrm{cM}$ on 14 linkage groups with one marker every $10.8 \mathrm{cM}$. Linkage groups on the two maps are temporarily assigned numbers according to their relative linkage distances. These do not necessarily correspond to chromosomes numbered according to length cytogenetically (Oliver, 1977). RAPD markers are indicated by the name of the Operon primer, a period followed by the size of the amplified fragment. A STAR locus is indicated by a RAPD label followed by an 'ST'. cDNA loci follow the labels designated in Valenzuela et al. (2002). Microsatellite loci are indicated by AC, AG, CTGY, or GATA and the clone number.

I. scapularis genome, then the relationship of physical to genetic distance is $\sim 267-329 \mathrm{~kb} / \mathrm{cM}$. Work is currently underway to determine the physical size and organization of the I. scapularis genome.

\section{Discussion}

Our experience in generating this preliminary linkage map demonstrates both promising aspects to and pitfalls for future work in tick genetics and genomics. Having tried conventional RAPDs, STARs, MS, and cDNAs, it appears that STARs are likely to be the marker of choice for expanding the I. scapularis linkage map and eventually integrating the linkage map into a physical map. Of the 65 STAR primer sets designed (Table 2), 52 were polymorphic and genotypes at 37 of these conformed to expected Mendelian ratios. The 
numbers of RAPD loci and the amount of heterozygosity at these loci were not as great as those detected in A. aegypti (Antolin et al., 1996). From the 20 RAPD primers, a total of 63 markers were mapped to the I. scapularis genome while 94 markers were generated with only 10 RAPD primers in an Ae. aegypti $\mathrm{F}_{1}$ intercross family (Antolin et al., 1996). Reassociation kinetic studies of another ixodid tick, A. americanum, showed that the organization of repetitive DNA was of the long-period interspersion type (Palmer et al., 1994). We suspect that this is also the case for I. scapularis.

Fagerberg et al. (2001) had to use a specialized capture technique to identify a few MS loci in I. scapularis. Furthermore, the heterozygosity at these few loci was low. We have no explanation for the low abundance and variability at MS loci in I. scapularis. Sequences obtained from cDNA libraries were useful in generating PCR primers that amplified single nucleotide polymorphisms (SNPs) for linkage mapping in an A. aegypti $\mathrm{F}_{1}$ intercross family (Fulton et al., 2001). In most cases the amplified regions were of the same size as the cDNA. However, this was not the case with cDNAs in the I. scapularis genome. In only 5 of the $20 \mathrm{cDNAs}$, for which primers were designed, were we able to recover a fragment that was sufficiently short for SSCP analysis. This appears to be due to the presence of large intervening introns. Taking the additional steps required to identify the locations and sequences of intron/exon boundaries will be very laborious and expensive.

We are in the process of performing the reassociation kinetics of the I. scapularis genome. However, assuming that the physical size of the I. scapularis genome is approximately the same size as the A. americanum genome $\left(1.04 \times 10^{9} \mathrm{bp}\right)($ Palmer et al., 1994), perhaps an inappropriate assumption to make, our results suggest that there is a great deal of recombination in I. scapularis. A resolution of $\sim 300 \mathrm{~kb} / \mathrm{cM}$ compares favorably to the average $1100 \mathrm{~kb} / \mathrm{cM}$ resolution in A. gambiae (Dimopoulos et al., 1996) or the 1000-3400 kb resolution for A. aegypti (Brown et al., 2001). A map of this resolution has a higher probability of being successfully used in mappedbased positional cloning of candidate genes for vector competence to pathogens, host preference, insecticide resistance and other important characters in I. scapularis.

\section{References}

Antolin, M.F., Bosio, C.F., Cotton, J., Sweeney, W., Strand, M.R. and Black, W.C.t. 1996. Intensive linkage mapping in a wasp (Bracon hebetor) and a mosquito (Aedes aegypti) with single-strand conformation polymorphism analysis of random amplified polymorphic DNA markers. Genetics 143: 1727-1738.

Black, W.C. and DuTeau, N.M. 1997. In: The Molecular Biology of Insect Disease Vectors: A Methods Manual, pp. 361-373. Chapman \& Hall, New York. 
Bosio, C.F., Fulton, R.E., Salasek, M.L., Beaty, B.J. and Black, W.C.t. 2000. Quantitative trait loci that control vector competence for dengue-2 virus in the mosquito Aedes aegypti. Genetics 156: 687-698.

Brown, S.E., Severson, D.W., Smith, L.A. and Knudson, D.L. 2001. Integration of the Aedes aegypti mosquito genetic linkage and physical maps. Genetics 157: 1299-1305.

Dietrich, W., Katz, H., Lincoln, S.E., Shin, H.S., Friedman, J., Dracopoli, N.C. and Lander, E.S. 1992. A genetic map of the mouse suitable for typing intraspecific crosses. Genetics 131: 423-447.

Dimopoulos, G., Zheng, L., Kumar, V., della Torre, A., Kafatos, F.C. and Louis, C. 1996. Integrated genetic map of Anopheles gambiae: use of RAPD polymorphisms for genetic, cytogenetic and STS landmarks. Genetics 143: 953-960.

Fagerberg, A.J., Fulton, R.E. and Black, W.C. 2001. Microsatellite loci are not abundant in all arthropod genomes: analyses in the hard tick, Ixodes scapularis and the yellow fever mosquito, Aedes aegypti. Insect Mol. Biol. 10: 225-236.

Fulton, R.E., Salasek, M.L., DuTeau, N.M. and Black, W.C.t. 2001. SSCP analysis of cDNA markers provides a dense linkage map of the Aedes aegypti genome. Genetics 158: 715-726.

Hayashi, K. 1991. PCR-SSCP: a simple and sensitive method for detection of mutations in the genomic DNA. PCR Meth. Appl. 1: 34-38.

Hiss, R.H., Norris, D.E., Dietrich, C.H., Whitcomb, R.F., West, D.F., Bosio, C.F., Kambhampati, S., Piesman, J., Antolin, M.F. and Black, W.C.t. 1994. Molecular taxonomy using single-strand conformation polymorphism (SSCP) analysis of mitochondrial ribosomal DNA genes. Insect Mol. Biol. 3: 171-182.

Hudson, T.J., Stein, L.D., Gerety, S.S., Ma, J., Castle, A.B., Silva, J., Slonim, D.K., Baptista, R., Kruglyak, L., Xu, S.H. et al. 1995. An STS-based map of the human genome. Science 270: $1945-1954$.

Hunt, G.J. and Page Jr., R.E. 1995. Linkage map of the honey bee, Apis mellifera, based on RAPD markers. Genetics 139: 1371-1382.

Kosambi, D.D. 1944. The estimation of map distances from recombination values. Ann. Eugen. 12: 172-175.

Oliver Jr., J.H. 1977. Cytogenetics of mites and ticks. Annu. Rev. Entomol. 22: 407-429.

Orita, M., Suzuki, Y., Sekiya, T. and Hayashi, K. 1989. Rapid and sensitive detection of point mutations and DNA polymorphisms using the polymerase chain reaction. Genomics 5: 874-879.

Palmer, M.J., Bantle, J.A., Guo, X. and Fargo, W.S. 1994. Genome size and organization in the ixodid tick Amblyomma americanum (L.). Insect Mol. Biol. 3: 57-62.

Sonenshine, D.E. 1991. Biology of Ticks, Vol. 1, New York.

Spielman, A. 1976. Human babesiosis on Nantucket Island: transmission by nymphal Ixodes ticks. Am. J. Trop. Med. Hyg. 25: 784-787.

Spielman, A., Wilson, M.L., Levine, J.F. and Piesman, J. 1985. Ecology of Ixodes damminiborne human babesiosis and Lyme disease. Annu. Rev. Entomol. 30: 439-460.

Stam, P. and van Oojin, J.W. 1995. CPRO-DLO, Wageningen, The Netherlands.

Telford III, S.R., Dawson, J.E., Katavolos, P., Warner, C.K., Kolbert, C.P. and Persing, D.H. 1996. Perpetuation of the agent of human granulocytic ehrlichiosis in a deer tick-rodent cycle. Proc. Natl. Acad. Sci. USA 93: 6209-6214.

Ullmann, A.J., Piesman, J., Dolan, M.C. and Iv, W.C. 2003. A preliminary linkage map of the hard tick, Ixodes scapularis. Insect Mol. Biol. 12: 201-210.

van Ooijen, J.W. 1994. DrawMap: a computer program for drawing genetic linkage maps. J. Hered. 85: 66. 
Vidal-Puig, A. and Moller, D.E. 1994. Comparative sensitivity of alternative single-strand conformation polymorphism (SSCP) methods. Biotechniques 17: 490-492, 494, 496.

Williams, J.G., Kubelik, A.R., Livak, K.J., Rafalski, J.A. and Tingey, S.V. 1990. DNA polymorphisms amplified by arbitrary primers are useful as genetic markers. Nucl. Acids Res. 18: 6531-6535.

Yasukochi, Y. 1998. A dense genetic map of the silkworm, Bombyx mori, covering all chromosomes based on 1018 molecular markers. Genetics 150: 1513-1525.

Zheng, L., Benedict, M.Q., Cornel, A.J., Collins, F.H. and Kafatos, F.C. 1996. An integrated genetic map of the African human malaria vector mosquito, Anopheles gambiae. Genetics 143: 941-952. 